\title{
Adubos de liberação lenta em cana-de-açúcar
}

\author{
Slow release fertilizers in sugarcane \\ M. E. Geromel ${ }^{1}$; C. E. Pereira ${ }^{2 *}$; A. L. P. Kikuti ${ }^{3}$; H. Kikuti ${ }^{4}$; J. R. Silva ${ }^{1}$ \\ ${ }^{1}$ Universidade Estadual de Mato Grosso do Sul/Unidade Universitária de Cassilândia \\ ${ }^{2}$ Universidade Federal do Sul da Bahia/Centro de Formação em Ciências Agroflorestais \\ ${ }^{3}$ Instituto Federal de Ciência e Tecnologia do Triângulo Mineiro \\ ${ }^{4}$ Universidade Federal de Uberlândia/Instituto de Ciências Agrárias \\ *ce-pereira@bol.com.br \\ (Recebido em 25 de setembro de 2018; aceito em 17 de junho de 2019)
}

\begin{abstract}
A utilização de adubos de liberação lenta permite que os nutrientes presentes no fertilizante estejam disponíveis quando realmente são necessários, diminuindo suas perdas e, consequentemente, possibilitando a redução das doses aplicadas. Neste sentido, avaliou-se o comportamento de duas variedades de cana-deaçúcar adubadas com $\mathrm{N}$ e $\mathrm{K}$ revestidos. Foram avaliadas a viabilidade de gemas, o número de perfilhos, a altura de plantas, a biomassa fresca e seca da parte aérea das plantas e o comprimento e produtividade de colmos industrializáveis. $\mathrm{O}$ uso de adubos $\mathrm{N}$ e $\mathrm{K}$ revestidos possibilita a redução de $50 \%$ da dose recomendada para o fertilizante convencional, sem afetar o crescimento e desenvolvimento da cana-deaçúcar.

Palavras-chave: Saccharum, crescimento, produtividade.
\end{abstract}

The use of slow release fertilizers allows the nutrients present in the fertilizer to be available when they are needed, reducing their losses and, consequently, reducing the applied doses. In this sense, was evaluated the behavior of two varieties of sugar cane fertilized with $\mathrm{N}$ and $\mathrm{K}$ coated. We evaluated the buds viability, number of tillers, plant height, and fresh and dry biomass of the shoots, the length and productivity or industrialized stalks. The use of fertilizer $\mathrm{N}$ and $\mathrm{K}$ coated allows the reduction of $50 \%$ of the recommended dose for conventional fertilizer, without affecting the growth and development of sugarcane.

Keywords: Saccharum, growth, productivity.

\section{INTRODUÇÃO}

As maiores extrações de nutrientes pela cultura da cana de açúcar ocorrem para potássio (K) e nitrogênio $(\mathrm{N})$, respectivamente [1], de modo que a resposta à adubação com estes elementos é sempre alta.

O teor de potássio disponível no solo, para crescimento e desenvolvimento da cana de açúcar, é um fator determinante da produtividade da lavoura, pois este desempenha na planta funções metabólicas e estruturais. Estima-se que para cada $100 \mathrm{t}$ de colmos, são exportados cerca de $125 \mathrm{~kg}$ de K [2]. Como a disponibilidade de potássio nos solos brasileiros normalmente é reduzida tornase necessário a aplicação de fertilizantes para obtenção de produtividades elevadas. Neste sentido, Otto et al. (2010) [3] constataram produtividade máxima de colmos de $160 \mathrm{t} \mathrm{ha}^{-1}$ para a aplicação de $150 \mathrm{~kg} \mathrm{ha}^{-1}$ de $\mathrm{K}_{2} \mathrm{O}$, sem parcelamento.

Devido à alta mobilidade do potássio no solo, perdas por lixiviação podem ocorrer, reduzindo, consequentemente, a disponibilidade deste elemento para as plantas. Como nas lavouras de cana a aplicação de potássio geralmente é realizada em dose única, variando de 80 a $140 \mathrm{~kg} \mathrm{ha}^{-1}$ de $\mathrm{K}_{2} \mathrm{O}$ [4], podem ocorrer perdas significativas do fertilizante aplicado dependendo da quantidade de chuva e da textura do solo, dentre outros fatores [5].

Quanto à adubação nitrogenada, tem-se observado uma resposta linear crescente em relação à produtividade de colmos, quando de sua aplicação na segunda ou terceira socas [6], sendo considerado também um elemento determinante na longevidade da cultura da cana [7]. Entretanto, 
na implantação do canavial a adubação nitrogenada pode ser dispensada ou, se necessário, aplicados até $30 \mathrm{~kg} \mathrm{ha}^{-1}$ de $\mathrm{N}$ [8].

Estima-se que, devido à ocorrência de perdas, a eficiência de aproveitamento de $\mathrm{N}$ pelas plantas seja de apenas $40 \%$ a $50 \%$ da quantidade aplicada. Neste sentido, visando reduzir perdas e aumentar a eficiência de uso do $\mathrm{N}$, tem-se realizado o controle da época de aplicação, melhoria nos métodos de aplicação, e, mais recentemente, o uso de revestimentos nos fertilizantes para controlar a liberação dos nutrientes.

Neste contexto, a utilização de fertilizantes que apresentam uma liberação mais lenta ou controlada, proporcionando a liberação dos nutrientes de acordo com os estádios de desenvolvimento e crescimento das plantas, tem sido considerada uma alternativa para aplicação mais eficiente de fertilizantes.

Os nutrientes encapsulados por resinas especiais, os quais são liberados através de estrutura porosa, atingem o sistema radicular das plantas mais lentamente [9]. Essa característica pode garantir a manutenção de um sincronismo entre a liberação de nutrientes ao longo do tempo e as necessidades nutricionais, favorecendo o crescimento e o desenvolvimento das plantas. Além disso, por meio dos fertilizantes polimerizados há uma diminuição das perdas de nutrientes por lixiviação, volatilização e fixação, possibilitando a redução da dose aplicada [10]. Neste sentido, pesquisas recentes têm apontado resultados promissores em relação ao uso de fertilizantes de liberação controlada $[11,12,13,14]$.

Outro importante fator de produção é o ambiente de cultivo, que consiste no agrupamento das interações dos atributos dos solos com as condições climáticas regionais [15]. Portanto, ambiente de produção é o somatório das interações entre os atributos de superfície, subsuperfície e grau de declividade dos solos associadas com o clima local. Deve-se considerar que a profundidade do solo está diretamente relacionada com a água disponível e com o volume de solo ocupado pelas raízes; a fertilidade, com a fonte nutricional das plantas; a textura relacionada com a quantidade de matéria orgânica, capacidade de troca de cátions e disponibilidade hídrica; e a água associada com a solução do solo, onde as plantas retiram seus nutrientes [16].

Os ambientes de produção de cana-de-açúcar da região Centro-Sul do Brasil, considerando a água disponível como o principal componente desses ambientes e, secundariamente, as condições químicas do solo, são divididos em 10 (A1, A2, B1, B2, C1, C2, D1, D2, E1 e E2). O ambiente A1 é aquele com capacidade de produzir maior quantidade de cana por hectare, e o E2 aquele com menor capacidade de produção [16].

Levando em consideração a importância da adubação com N e K para o desenvolvimento e produtividade da cana-de-açúcar e a perda de $\mathrm{N}$ e $\mathrm{K}$ por lixiviação, objetivou-se avaliar o crescimento, desenvolvimento e produtividade de variedades de cana-de-açúcar em função de adubações de plantio com $\mathrm{N}$ e $\mathrm{K}$ revestidos com polímeros.

\section{MATERIAL E MÉTODOS}

A pesquisa foi realizada na área experimental da Universidade Estadual de Mato Grosso do Sul (UEMS), Unidade Universitária de Cassilândia - MS, em uma altitude de $470 \mathrm{~m}$, localizada a $18^{\circ} 59^{\prime} 44^{\prime \prime} \mathrm{S} ; 52^{\circ} 21^{\prime} 56^{\prime}$ 'O, com pluviosidade média anual de $1.500 \mathrm{~mm}$ e temperatura média de 32 ${ }^{\circ} \mathrm{C}$. O solo foi classificado como Latossolo Vermelho Amarelo de textura arenosa.

A caracterização dos atributos químicos foi realizada por meio de amostras coletadas na camada 0-20 cm do solo, obtendo-se o seguinte resultado: $\mathrm{pH}\left(\mathrm{H}_{2} \mathrm{O}\right) 4,8 ; 15 \mathrm{~g} \mathrm{dm}^{-3} \mathrm{de} \mathrm{MO} ; 5 \mathrm{mg} \mathrm{dm}^{-3} \mathrm{de}^{-3}$ P; 22 mmol $_{\mathrm{c}} \mathrm{dm}^{-3} \mathrm{de} \mathrm{H}+\mathrm{Al} ; 0,9 \mathrm{mmol}_{\mathrm{c}} \mathrm{dm}^{-3} \mathrm{de} \mathrm{K}_{1} ; 4 \mathrm{mmol}_{\mathrm{c}} \mathrm{dm}^{-3} \mathrm{de} \mathrm{Ca} ; 5 \mathrm{mmol}_{\mathrm{c}} \mathrm{dm}^{-3} \mathrm{de} \mathrm{Mg} ; 19,9$ mmol $_{\mathrm{c}} \mathrm{dm}^{-3}$ de SB; 41,9 mmol $_{\mathrm{c}} \mathrm{dm}^{-3}$ de CTC; $0,70 \mathrm{mg} \mathrm{dm}^{-3} \mathrm{de} \mathrm{Cu} ; 22 \mathrm{mg} \mathrm{dm}^{-3}$ de Fe; 4,4 mg dm${ }^{-3}$ de $\mathrm{Mn} ; 0,6 \mathrm{mg} \mathrm{dm}^{-3} \mathrm{de} \mathrm{Zn} ; 0,19 \mathrm{mg} \mathrm{dm}^{-3} \mathrm{de} \mathrm{B} ; 47,5 \%$ de saturação por bases (V); 0,0\% de saturação por alumínio (m); e também na camada de 20-40 cm: $\mathrm{pH}\left(\mathrm{H}_{2} \mathrm{O}\right) 4,7 ; 8 \mathrm{~g} \mathrm{dm}^{-3}$ de $\mathrm{MO} ; 3,0 \mathrm{mg} \mathrm{dm}^{-3}$ de P; $20 \mathrm{mmol}_{\mathrm{c}} \mathrm{dm}^{-3}$ de $\mathrm{H}+\mathrm{Al} ; 1,1 \mathrm{mmol}_{\mathrm{c}} \mathrm{dm}^{-3} \mathrm{de} \mathrm{K} ; 12 \mathrm{mmol}_{\mathrm{c}} \mathrm{dm}^{-3} \mathrm{de} \mathrm{Ca} ; 4 \mathrm{mmol}_{\mathrm{c}} \mathrm{dm}^{-3} \mathrm{de} \mathrm{Mg}$; 
$17,1 \mathrm{mmol}_{\mathrm{c}} \mathrm{dm}^{-3}$ de soma de bases (SB); 37,1 $\mathrm{mmol}_{\mathrm{c}} \mathrm{dm}^{-3} \mathrm{de} \mathrm{CTC} ; 0,60 \mathrm{mg} \mathrm{dm}^{-3} \mathrm{de} \mathrm{Cu} ; 12 \mathrm{mg} \mathrm{dm}^{-}$ ${ }^{3}$ de Fe; $2,2 \mathrm{mg} \mathrm{dm}^{-3}$ de $\mathrm{Mn} ; 0,3 \mathrm{mg} \mathrm{dm}^{-3}$ de $\mathrm{Zn} ; 0,11 \mathrm{mg} \mathrm{dm}^{-3}$ de B; 46,1\% de saturação por bases (V); $0,0 \%$ de saturação por alumínio (m).

As parcelas foram compostas de quatro fileiras de plantas, com sete metros de comprimento e espaçamento de 1,5 m entre sulcos. A área útil de cada parcela foi constituída das duas fileiras centrais de seis metros de comprimento. Foi realizado o preparo convencional do solo, com arações e gradagens. Foi aplicado o equivalente a $1,5 \mathrm{t} \mathrm{ha}^{-1}$ de calcário dolomítico, seguido de incorporação e 2 meses em pousio, sendo posteriormente sulcada a profundidade de $0,40 \mathrm{~m}$.

A adubação mineral de base com N e K, foi realizada utilizando-se $100 \%$ da recomendação de adubação baseada em uma produtividade $<100 \mathrm{t} \mathrm{ha}^{-1}$, sem revestimento com polímeros (Tratamento 1), utilizando $30 \mathrm{~kg} \mathrm{ha}^{-1}$ de $\mathrm{N}$ (fonte uréia) e $80 \mathrm{~kg} \mathrm{ha}^{-1} \mathrm{de} \mathrm{K}_{2} \mathrm{O}$ (fonte cloreto de potássio), e $50 \%$ da recomendação da adubação mineral de base com $\mathrm{N}$ e $\mathrm{K}$ revestidos com polímeros (Tratamento 2), utilizando $15 \mathrm{~kg} \mathrm{ha}^{-1}$ de $\mathrm{N}$ (fonte uréia), e $40 \mathrm{~kg} \mathrm{ha}^{-1}$ de $\mathrm{K}_{2} \mathrm{O}$ (fonte cloreto de potássio). Em

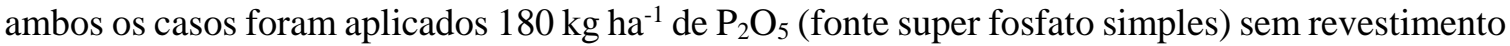
de polímeros, também, seguindo $100 \%$ da recomendação de adubação baseada em uma produtividade $<100 \mathrm{t} \mathrm{ha}^{-1}$.

Foram utilizadas duas variedades de cana-de-açúcar, RB867515 e RB72454, sendo os toletes colhidos manualmente no distrito de Vila Raimundo no município de Cassilândia. No plantio os toletes foram dispostos no sentido "pé com ponta", objetivando um número médio de 15 gemas viáveis por metro de sulco de plantio.

Foram realizadas as avaliações de viabilidade de gema (contagem das brotações na área útil, aos 15 dias após o plantio (DAP) e aos 30 DAP); número de perfilhos (contagem do número de perfilhos por metro, na área útil aos 45, 65, 85, 105 e 205 DAP); altura das plantas (após o surgimento da folha +1 (75 DAP), com o auxílio de uma régua graduada em $\mathrm{cm}$, medindo-se a altura da planta do colo até a folha +1 [17], sendo que as plantas foram marcadas para serem novamente avaliadas aos $95,115,205,265$ e 375 DAP).

Foi avaliada também a biomassa fresca e seca da parte aérea aos 115 DAP, coletando-se, ao acaso, três plantas por parcela, determinando-se a biomassa fresca. As plantas foram em seguida secadas em estufa com circulação de ar forçada, onde permaneceram por 72 horas à $65{ }^{\circ} \mathrm{C}$, avaliando-se após este período a biomassa seca. O comprimento de colmos industrializáveis foi determinado por meio da medição do comprimento da base da planta colhida até o local de corte, no ápice, realizado por ocasião da colheita. A produtividade de colmos industrializáveis foi avaliada com a determinação da massa de colmos na área útil de cada parcela, fazendo uso de balança digital.

$\mathrm{O}$ delineamento utilizado foi blocos casualizados em esquema fatorial $2 \times 2$, com 4 repetições, sendo 2 tipos de material propagativo (variedades RB867515 e RB72454) e 2 adubações com N e K (100\% da recomendação com adubo não revestido e 50\% da recomendação com adubos revestidos com polímero). Para as variáveis número de perfilho e altura de plantas, foi incluído no esquema experimental o fator épocas de avaliação, sendo 5 épocas para avaliação do número de perfilhos e 6 para altura de plantas, dando origem aos fatoriais $2 \times 2 \times 5$ e $2 \times 2 \times 6$, respectivamente.

Os dados foram submetidos a análise de variância utilizando-se o pacote computacional SISVAR [18]. As médias dos fatores variedades e adubos foram comparadas por meio do teste de F. As diferentes épocas de avaliação do número de perfilhos e da altura de planta foram estudadas por meio da análise de regressão.

\section{RESULTADOS E DISCUSSÃO}

Pelos resultados obtidos na análise de variância, verificou-se que as variedades RB867515 e RB72454, não diferiram significativamente entre si por meio da avaliação de viabilidade de gemas aos 15 dias após o plantio (Tabela 1). Foi verificada diferença significativa entre as variedades de cana-de-açúcar para a viabilidade de gemas aos 30 dias, biomassa fresca e seca da parte aérea, bem como para a altura de plantas. 
Tabela 1. Médias de viabilidade de gemas por metro de sulco aos 15 (VG15) e 30 (VG30) dias após o plantio - DAP, biomassa fresca (BF) e seca (BS) da parte aérea e altura das variedades RB867515 e RB72454.

\begin{tabular}{lccccc}
\hline \multirow{2}{*}{ Variedades } & \multicolumn{5}{c}{ Parâmetros Avaliados } \\
\cline { 2 - 6 } & VG15 & VG30 & BF (t ha $\left.\mathbf{~}^{-1}\right)$ & BS (t ha \\
\hline RB 867515 & $5,725 \mathrm{a}$ & $14,363 \mathrm{a}$ & $25,940 \mathrm{a}$ & $7,875 \mathrm{a}$ & $115,74 \mathrm{a}$ \\
RB 72454 & $4,638 \mathrm{a}$ & $11,100 \mathrm{~b}$ & $19,907 \mathrm{~b}$ & $5,835 \mathrm{~b}$ & $108,44 \mathrm{~b}$ \\
\hline CV (\%) $\mathbf{~}^{\mathbf{1}}$ & 39,0 & 20,6 & 20,2 & 24,7 & 8,57
\end{tabular}

Médias seguidas pela mesma letra, na coluna, não diferem estatisticamente pelo teste de $\mathrm{F}$ ao nível de 5\% de probabilidade. ${ }^{1}$ Coeficiente de variação.

Quanto à utilização de fertilizantes polimerizados, não foram verificadas diferenças significativas para a viabilidade gemas e biomassa da parte aérea. Assim, constatou-se que para estas variáveis a redução de $50 \%$ da dose de fertilizante aplicados, com uso do revestimento com polímeros foi vantajosa em relação à adubação com $100 \%$ da recomendação sem o revestimento com polímeros, pela economia de utilização dos fertilizantes.

O comprimento de colmos industrializáveis de cana-planta não apresentou diferenças relativas à utilização de adubos $\mathrm{N}$ e $\mathrm{K}$ com ou sem revestimento de polímeros, mesmo considerando as diferentes variedades, reforçando a constatação de ser a redução de $50 \%$ da recomendação da adubação com utilização de adubos $\mathrm{N}$ e $\mathrm{K}$ revestidos com polímeros, vantajosa em relação à adubação convencional sem a utilização do revestimento.

O comportamento de cada variedade em função do número de perfilhos e datas de avaliação são apresentadas na Figura 1. Destaca-se a variedade RB867515, com maior número de perfilhos por metro de sulco até 105 DAP, ou seja, intenso perfilhamento inicial.

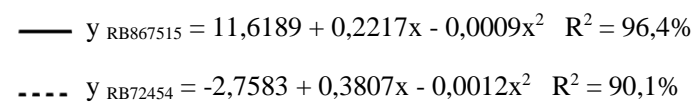

$y=-57,368+0,908 x \quad R^{2}=90,6 \%$
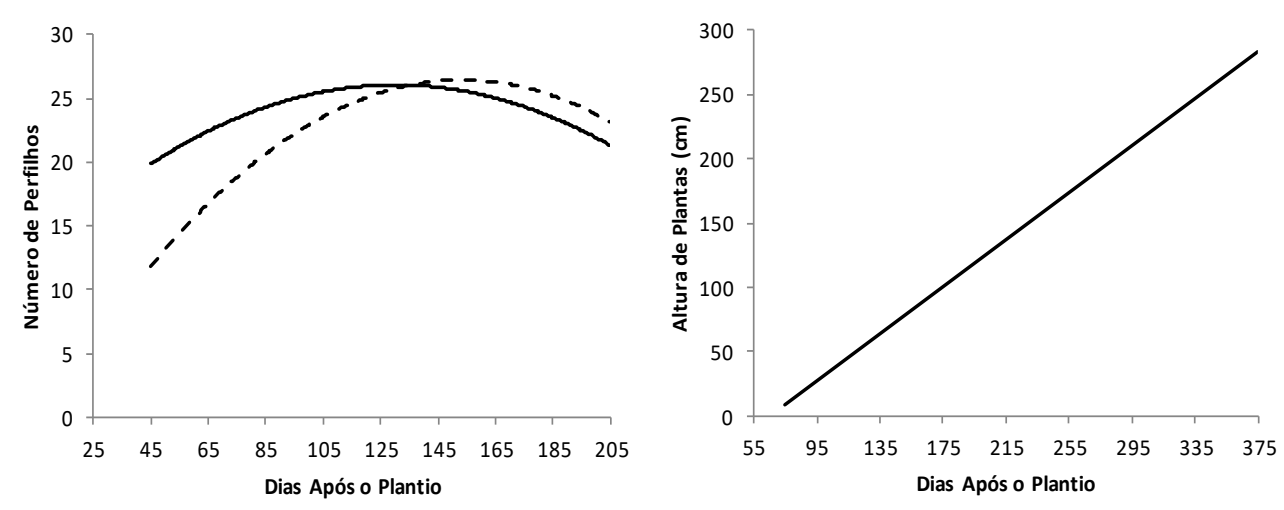

Figura 1. Número de perfilhos por metro de sulco das variedades RB867515 e RB72454 e altura de plantas de cana-planta em diferentes épocas de avaliação após o plantio.

A variedade RB72454 apresentou resultados inferiores nas avaliações iniciais de perfilhos até cerca de 125 dias, destacando a influência das variedades no comportamento da cultura instalada a campo (Figura 1). Resultados similares também foram obtidos por Oliveira (2004) [19] estudando o perfilhamento em cana-planta, utilizando três variedades RB72454, RB855113 e RB855536. No trabalho citado todas as variedades estudadas apresentaram comportamentos diferentes entre si aos 
497 DAP, sendo o máximo de perfilhamento obtido na variedade RB855536 e o menor na variedade RB72454.

O número de perfilhos não foi influenciado pela redução de $50 \%$ da dose dos adubos recomendados, quando associado ao uso do polímero já que não foi observado efeito significativo da interação entre épocas de avaliação e o tipo de fertilizante utilizado para esta variável.

Quanto à altura das plantas, não houve diferenças entre as variedades, tendo apresentado, na média, crescimento linear significativo (Figura 1). Também para a altura de plantas, a utilização de $50 \%$ da dose recomendada de adubos com polímeros possibilitou, significativamente, maior crescimento das plantas de cana-de-açúcar $(114,49 \mathrm{~cm})$ que a utilização de $100 \%$ da dose recomendada sem revestimento (109,70). Brondani et al. (2008) [20] obtiveram adequada altura de plantas com a utilização de adubos polimerizados, em doses crescentes de fertilizantes de liberação controlada no crescimento inicial de mudas de angico-branco, com acréscimo de aproximadamente $16 \%$ em relação a testemunha (contendo substrato comercial a base de casca de pinus e vermiculita) na qual não foi adicionado fertilizante de liberação lenta.

Para a produtividade de colmos industrializáveis não houve diferença significativa entre os fertilizantes revestido e convencional, independentemente da variedade de cana utilizada (Tabela 2).

Tabela 2. Valores médios de produtividade ( $\left.t h a^{-1}\right)$ de variedades de cana-de-açúcar RB 867515 e RB 72454 em função da utilização de adubos com ou sem polímeros.

Variedades

Produtividade $\left(\mathrm{t} \mathrm{ha}^{-1}\right)$

\begin{tabular}{lcc}
\cline { 2 - 3 } & Com Polímero & Sem Polímero \\
\hline RB 867515 & $55,90 \mathrm{aA}$ & $48,86 \mathrm{aA}$ \\
RB 72454 & $41,95 \mathrm{aA}$ & $48,75 \mathrm{aA}$
\end{tabular}

$\mathrm{CV}(\%)^{\mathbf{1}} 17,0$

Médias seguidas pela mesma letra minúscula na coluna ou maiúscula na linha não diferem estatisticamente entre si pelo teste de F, respectivamente, ao nível de 5\% de probabilidade. ${ }^{1}$ Coeficiente de Variação.

Otto et al. (2010) [3] concluíram em seu trabalho que a produtividade máxima estimada da canade-açúcar poderia ser obtida com a aplicação de $130 \mathrm{~kg} \mathrm{ha}^{-1}$ de $\mathrm{K}_{2} \mathrm{O}$ na forma parcelada ou $150 \mathrm{~kg}$ $\mathrm{ha}^{-1}$ de $\mathrm{K}_{2} \mathrm{O}$ de uma única vez no plantio, evidenciando que uma disponibilidade gradual do potássio, mesmo em doses menores, poderia favorecer a produção da planta. De modo semelhante, a aplicação dos adubos associados ao tratamento com polímero, pode propiciar maior economia com fertilizantes devido a sua liberação gradual, reduzindo as perdas no solo, já que os fertilizantes potássicos e nitrogenados são facilmente lixiviados [5].

\section{CONCLUSÃ̃o}

O uso de adubos de liberação lenta permitiu uma redução de $50 \%$ da adubação básica de $\mathrm{N}$ e K, sem prejuízo ao crescimento e desenvolvimento inicial da cana-de-açúcar, nas condições deste trabalho.

\section{REFERÊNCIAS BIBLIOGRÁFICAS}

1. Orlando Filho J, Zambello Junior E. Influência da adubação NPK nas qualidades tecnológicas da cana planta, variedade CB41-76. Brasil Açucareiro 1980;96(4):37-44.

2. Malavolta E. Potássio e enxofre nos solos e culturas brasileiras. Piracicaba, Potafós, 1982. (Boletim Técnico, 4). 
3. Otto R, Vitti GC, Luz PHC. Manejo da adubação potássica na cultura da cana-de-açúcar. Rev Bras Ciênc Solo. 2010 Jul/Ago;34(4):1137-1145, doi: 10.1590/S0100-0683201000040001.

4. Lana RMQ, Zanão Júnior LA, Korndorfer GH, Maciel Junior VA. Parcelamento da adubação potássica na cana-planta. STAB: Açúcar, Álcool e Subprodutos. 2004;23(2):28-31.

5. Rosolem CA, Santos FP, Foloni JSS, Calonego JC. Potássio no solo em consequência da adubação sobre a palha de milheto e chuva simulada. Pesq Agropec Bras. 2006 Jun;41(6):1033-1040, doi: 10.1590/S0100-204X2006000600020.

6. Vitti AC, Trivelin PCO, Gava GJC, Penatti CP, Bologna IR, Faroni CE, Franco HCJ. Produtividade da cana-de-açúcar relacionada ao nitrogênio residual da adubação e do sistema radicular. Pesq Agropec Bras. 2007 Fev;42(2):249-256, doi: 10.1590/S0100-204X2007000200014.

7. Franco HCJ, Bologna IR, Faroni CE, Vitti AC, Trivelin PCO. Acúmulo de macronutrientes em cana-deaçúcar em função da adubação nitrogenada e dos resíduos culturais incorporados ao solo no plantio. Bragantia 2007 Mai;66(4):669-674, doi: 10.1590/S0006-87052007000400017

8. Boddey RM, Polidoro JC, Resende AS, Alves BJR, Urquiaga S. Use of the $15 \mathrm{~N}$ natural abundance technique for the quantification of the contribution of $\mathrm{N} 2$ fixation to sugar cane and other grasses. Aust $\mathrm{J}$ Plant Physiol. 2001 Sept;28(1):1-7, doi: 10.1071/PP01058.

9. Hanafi MM, Eltaib SM, Ahmad MB. Physical and chemical characteristics of controlled release compound fertilizer. Eur Polym J. 2000 Oct;36(10):2081-2088, doi: 10.1016/S0014-3057(00)00004-5.

10. Zahrani S. Utilization of polyethylene and paraffin waxes as controlled delivery systems for different fertilizers. Ind Eng Chem Res. 2000 Dec;39(2):367-371, doi: 10.1021/ie980683f.

11. Guareschi RF, Gazolla PR, Perin A, Santini JMK. Adubação antecipada na cultura da soja com superfosfato triplo e cloreto de potássio revestidos por polímeros. Ciênc Agrotec. 2011 Jan;35(4):643648, doi: 10.1590/S1413-70542011000400001.

12. Souza CHE. Fosfato monoamônio revestido com polímeros no plantio das culturas de milho irrigado e cana-de-açúcar [tese]. Uberlândia (MG): Universidade Federal de Uberlândia; 2012. 81 p.

13. Guareschi RF, Perin A, Gazolla PR. Produtividade de milho submetido à aplicação de ureia revestida por polímeros. Global Sci Techn. 2013 Mai/Ago;6(2):31-37, doi: 10.14688/1984-3801.V06N02A04.

14. Martins IS. Doses, épocas e modos de aplicação da uréia comum e revestida na cultura do milho [dissertação]. Jaboticabal (SP): Universidade Estadual Paulista; 2013. 63 p.

15. Ripoli TCC, Ripoli MLZ, Casagrandi DV, Ide BY. Plantio de Cana-de-açúcar: estado da arte. 2 ed. Revisão completa. Piracicaba (Brasil), 2007. p. 60-96.

16. Prado H. Ambientes de produção de cana-de-açúcar na região Centro-Sul do Brasil. Potafós, Piracicaba. Informações Agronômicas 2005 110:12-17. (Encarte Técnico).

17. Mozambani AE, Pinto AS, Segato SV, Mattiuz CFM. História e morfologia da cana-de-açúcar. In: Segato SV, Pinto AS, Jendiroba E, Nóbrega JCM. (Org.) Atualização em Produção de Cana-de-Açúcar. Piracicaba: Livroceres, 2006, p. 15.

18. Ferreira DF. Sisvar: a Guide for its Bootstrap procedures in multiple comparisons. Ciênc Agrotec. 2014 Mar/Apr;38(2):109-112, doi: 10.1590/S1413-70542014000200001.

19. Oliveira RA. Análise de Crescimento de Cana-de-Açúcar, na Região Noroeste do Paraná [dissertação]. Curitiba (PR): Universidade Federal do Paraná Agronomia; 2004. 65 f.

20. Brondani GE, Silva AJC, Rego SS, Grisi FA, Nogueira I, Araújo MA. Fertilização de liberação controlada no crescimento inicial de angico-branco. Scientia Agraria. 2008;9(2):167-176, doi: 10.5380/rsa.v9i2.10965. 